\title{
Decoherence And Ontology
}

\section{Roland Omnès}

Faculté des sciences at Orsay

Université Paris-Sud XI

In the year 2000, exactly one century after the discovery of quanta, it is very remarkable that no change was found necessary in the basic principles of quantum mechanics, which remain the same as when stated in the late Twenties. The situation in interpretation and epistemology is still fluid, on the contrary. There is now a trend among philosophers to refuse the drastic revision in the philosophy of knowledge that was thought necessary after the work by Bohr, Heisenberg and Pauli (and still is in my opinion). Together with a few physicists, these philosophers nurture the hope of seeing a new realistic theory come out, although there is no sign of a complete or consistent one yet. A very different trend of research existing among physicists might be described on the other hand as being both pragmatic and theoretical, two characteristics which are apparently opposite and require therefore an explanation.

When I say that our understanding of quantum mechanics is now completely pragmatic, I mean that every concept in it, either important or tiny, is not only appreciated as a building block in a grand construction, but as an individual piece of knowledge expressing directly the results of some specially dedicated experiments. The present experimental techniques are so powerful that the investigation of an aspect or another of the quantum world has become a most enjoyable testing ground for the tools at our disposal. The paradigm of this approach goes back presumably to the experiment that was performed in 1982 by Alain Aspect for checking Bell's inequalities and resulting in an evidence for entangled states ${ }^{1}$. The emphasis has slightly changed however. Whereas John Bell asked a deep question, more akin to the structure of our understanding than anything else (such as locality or a causality hidden in hidden variables) ${ }^{2}$, the experimental result is now more or

1. A. Aspect, P. Grangier, G. Roger, Phys. Rev. Lett. 47, 460 (1981).

2. J. S. Bell, Physics 1, 195 (1964). 
less taken as meaning: "Never mind philosophical issues. Just think as Nature lets you to do it by giving you plain facts". The number of plain facts confirming quantum concepts and resulting from smart experiments has now tremendously increased and all of them, up to now, are in essential agreement with the Copenhagen rules.

When one tries to catch more of the spirit of this time (l'esprit du temps) among the majority of physicists, one does not always find them full adepts of the Copenhagen philosophy, in spite of their acceptance of the practical Copenhagen rules. The real existence of wave packet reduction, for instance, is often considered as remaining more or less an open question; the gap between classical and quantum physics and between determinism and probabilism, is considered as somewhat less important than their correspondence, particularly in the case of quantum-behaving macroscopic systems or mesoscopic ones ${ }^{3}$.

So, clearly, the present episteme is pragmatic. But it is also systematically theoretical. Theoreticians accept readily the basic principles of quantum mechanics, with no reservation, because these principles have withstood a tremendous widening of their range when extended from atoms to the standard model of particles, and without a hint of weakness. These basic laws and concepts consist of the Hilbert space framework incorporating non-commuting physical quantities, of a law for dynamics resulting from the Schrödinger equation and a few more assumptions among which relativistic invariance and the existence of identical particles are the main ones, since they are essential for the existence of quantum fields. Similarly, since a few decades, interpretation has also become more and more a topic of theoretical research, where one tries to extract from the basic principles themselves some new consequences allowing to deepen their understanding. Theoretical work or, essentially, mathematical investigation becomes accordingly some sort of a required preliminary before any further philosophical reflection.

I thought that such a brief description of the state of research would be useful when speaking to a majority of philosophically-trained people as we are here, if only because it is so different from the approach you may be used to. We shall see together however that philosophical questions are like the heads of the famous Hydra, always poking them from new unexpected directions.

The main pioneer of the present spirit among the founding fathers of quantum mechanics was certainly John von Neumann. Although he worked at a time when speculations, research programmes and personal convictions were not considered as suitable for publication, there is little doubt that he did not accept the double talk of using sometimes a classical language and sometimes a quantum one in the statement of physical laws. He does not seem either to have had much respect for the complementarity principle, probably too Hegelian for a logician such as him.

As a matter of fact, von Neumann began his career by doing research in logic, with the

3. J. Clarke, A. N. Cleland, M. Devoret, D. Esteve, J. M. Martinis, Science 239, 992 (1988). 
foundations of set theory, and his later work on the logic of computers is well known. It may be mentioned that he belonged to the school of thought headed by Hilbert, according to whom theoretical physics should rely on well-formulated basic axioms and then consist of their rigorously derived consequences. The famous book by von Neumann, Mathematische Grundlagen der Quantenmechanik, was a powerful attempt at satisfying these exacting requirements and one may remember how he pointed there Hilbert spaces as the right framework for quantum concepts, while improving considerably for that purpose the theory of operators.

Another important idea in that book was to define a proposition, a statement expressing a physical property of a quantum system, by "elementary predicates" according to which "the value of some observable A lies within a range Æ of real numbers at a time $t$ ". Indeed, it turns out that every statement concerning a quantum event can always be expressed by such a predicate. Furthermore, one can always translate the statement into the language of mathematics by associating it with a definite "projection operator", namely the mathematical operator projecting a state vector on a Hilbert subspace (consisting of the eigenstates of the operator $\mathrm{A}(\mathrm{t})$ with an eigenvalue in the range $Æ)$. An important consequence of this construction is to subject the logic of quantum properties to the rigor of mathematics, a paradigm to which von Neumann later gave another expression with the logic of computers. In both cases, there is emphasis on the Aristotelian principle according to which a proposition can only have two possible values: "true" or "false". In a computer, this is obtained by assigning the value of a proposition to some memory, which can either withstand a standard voltage for "true", or a zero voltage for "false". In quantum mechanics, a projection operator has the same two-valuedness since it can only have two eigenvalues, 1 or 0 .

With his projection operators, von Neumann had discovered something essential for the understanding of quantum mechanics, namely a language that could bridge the gap between the intuition of a pragmatic physicist (and also, why not, of a philosopher) and the formal requirements of a mathematician, since the concepts and laws of quantum physics can only be fully stated in mathematical terms. When however his book was published, the readers were much more impressed by three failures he had met, all of them of course duly acknowledged. One of these great difficulties was the prediction of macroscopic quantum interferences in the final state of a quantum measurement device, a result appearing in the last two pages of the book offering a quantum model for a measurement. This remark was later to become famous when explained by Schrödinger with the example of his unforgettable Cat.

There were two other difficulties, one of them with classical physics. Classical properties do not only state for instance that the position of a particle lies in some range of values, but that the position and the velocity of some macroscopic part of an apparatus can be assigned simultaneously some values, even with large enough errors allowing a minimal 
violation of the uncertainty relations. Although he made a nice try at it, von Neumann was not able to associate a projection operator with a classical property and his predicates could not be therefore considered at that time as providing a universal language for physics.

The last difficulty had to do with logic. If all the possible predicates, or all the possible projectors, express so many sensible propositions, then it is impossible to define the basic logical operations (not, and, or) and the corresponding relations (equivalence and inference) while satisfying the standard rules of logic. For a long time, this difficulty generated a trend of thought according to which the logic of quantum mechanics might be non-standard. Finally, it seemed that von Neumann's language for quantum mechanics was neither universal nor convincingly sensible, which is why it did not much influence interpretation for a long time.

Though rather old, this story remains certainly the best introduction to more recent research. It was first seen negatively: three outstanding difficulties stood on the way of a deductive interpretation of quantum mechanics. They looked so insuperable that von Neumann himself proposed almost incredible solutions, such as leaving to consciousness the burden of removing macroscopic superpositions. He also considered seriously the possibility of non-standard logic as a key to the understanding of quanta ${ }^{4}$. With hindsight, these somewhat desperate attempts show that the difficulties were really non-trivial but, from a positive point of view, it can also be said that they were well-defined problems, which held the key for a deeper interpretation.

The theoretical approach to interpretation has led to answers for the three von Neumann problems in the last two decades or so. For macroscopic interferences, the name of the answer is "decoherence". The precise derivation of classical physics from the quantum principles was obtained by three different methods using either "coarse graining" 5 , "coherent states" 6 or a newcomer in mathematics known as "microlocal analysis" "; it shows explicitly how classical determinism is a consequence of probabilistic quantum laws. Finally the problem of logic was solved by introducing "consistent histories" ${ }^{8}$, in which the propositions describing physical properties are not single predicates but socalled histories. When put together, the three answers lead easily to a completely deductive interpretation of quantum mechanics, in which the basic principles are enough for generating their own interpretation'. The usual rules of measurement theory for instance become so many theorems.

4. G. Birkhoff, J. von Neumann, Ann. Math. 37, 818 (1936).

5. M. Gell-Mann, J. B. Hartle, in Complexity, Entropy and the Physics of Information, W. H. Zurek (edit.), Addison-Wesley, Redwood City, CA (1991).

6. K. Hepp, Comm. Math. Phys. 35, 265 (1974)

7. R. Omnès, J. Stat. Phys. 57, 357 (1989) ; J. Math. Phys. 38, 697 (1997).

8. R. G. Griffiths, J. Stat. Phys. 36, 219 (1984).

9. R. Omnès, Understanding Quantum Mechanics, Princeton University Press (1999). 
My purpose in the present talk is not however to describe this interpretation in detail, because it would be too long, but to identify the new philosophical problems one is left with at the end. The most important new item is certainly decoherence. I shall not try to describe it technically, but only mention the essential ideas. When considering a macroscopic object, we perceive only some obvious collective degrees of freedom describing for instance the position and the shape of its various parts. We should not forget however that the object itself (for instance a detector) contains typically billions of billions of billions other degrees of freedom for all the atoms in it. The degrees of freedom of an external environment (atmospheric molecules around the object or photons in the surrounding light) can also play a role at the quantum level and, globally, it has become conventional to call the formal subsystem containing all these degrees of freedom (internal and external) the environment. The accessible (collective) degrees of freedom one can directly perceive and measure label, from a formal standpoint, another subsystem, both abstract mathematically and obvious empirically. The whole object is therefore considered formally as made of two systems, one we can see and one we cannot control in detail.

You may remember that Heisenberg already considered the environment (without the name) as opening a possible way out of the cat problem. This possibility has now been investigated in some detail and, basically, the following schedule is found: The two systems (collective and environment) are coupled. They can exchange energy as we know from the existence of friction and dissipation, so that a part of the total hamiltonian must connect them, couple them. Now I suppose that you do not easily envision a complex wave function of the environment depending on so many billions of variables no more than I do, but let us say that it is very complicated and, most importantly, extremely sensitive to the external coupling. When a wheel turns even so slightly in a clock, what it provokes in the environment wave function is a cataclysm: atoms move, electrons are shaken and phases, which are the most sensitive and delicate features of a wave function, change practically at random.

Suppose now that the wheel belongs to a measuring device, guiding for instance a voltmeter pointer whose position will indicate the actual result of a measurement. What happens ? According to Schrödinger and his cat or von Neumann and his mathematics, the final wave function of the measuring device is a sum of two terms, one indicating, say, that the pointer did not move and another according to which the final position of the pointer has turned by 90 degrees, indicating that something has been detected. But in fact, these two parts of the wave function are very different. Already when the wheel was beginning to move, the environment wave function, with its many billions of variables, was behaving very differently for the static wheel than it did for the moving one. The corresponding phases (i.e. a phase for every value of every variable in the crowd), their phases I say had soon lost any hint of coherence: they decohered, according to a useful neologism stamped out by Gell-Mann. 
In the example of the cat, one would say that the wave functions of the set of atoms inside a live cat and a dead one differ so much in their multitudinous phases that they cannot be anything but orthogonal: they do not allow any visible interference at the level of the cat body. The non-existence of macroscopic interferences which looked so troublesome has now an obvious origin: destructive interferences in the environment wave functions suppress constructive ones at the atomic level.

The theory of decoherence is of course more precise than the sketch I just gave ${ }^{10} 11{ }^{12}$. As it turns out, decoherence is in fact a special kind of irreversible process ${ }^{13}$. It is moreover an extraordinarily effective effect, so quick in action that it completely suppressed any interference before it could be spotted by an observation. And so, for many years, theorists have lived with a solution that no experiment could establish. Who would then believe equations when so much is at stake! But fortunately, four years ago, the effect was seen at last in an experiment of quantum optics, where the number of degrees of freedom in an environment could be made to vary from zero to ten. Interference were then seen to disappear, gradually, in exact agreement with theoretical predictions ${ }^{14}$. Something essential is therefore now established. Decoherence exists and it is as much effective as we did expect. So much then with physics. The next question should be to consider the kind of consequence it has for our understanding of physical reality ${ }^{15}{ }^{16}$.

It is certainly not a surprise if various people have very different reactions to the experimental discovery of decoherence. People believing in Bohmian mechanics do not care: they live in a world much above any experimental reach. People who are fond of actual reduction mechanisms recognise that decoherence does the job more rapidly and completely than any unconventional effect they had proposed for the same purpose. Some of them say that two effects are still better than one.

What about people who accept quantum mechanics as complete? It is very instructive for instance to put together the answers for the three von Neumann problems and draw the consequences. There are in fact so many of them that only a book can give their list but I can mention one: It is found that a definite direction of time comes out from three different origins: there is dissipation (with the second principle), decoherence (!) and also logic (leaving aside the cosmological direction of time). As far as logic is concerned, some histories for a quantum system make sense with standard logic with one direction of time but they do not with the opposite direction, when the film of events is run back. These

10. W. H. Zurek, Phys. Rev. D 26, 1862 (1982).

11. A. O. Caldeira, A. J. Leggett, Physica A 121, 587 (1983).

12. E. Joos, H. D. Zeh, Z. Phys. B 59, 229 (1985).

13. R. Omnès, Phys. Rev A 56, 3383 (1997).

14. M. Brune et al. Phys. Rev. Lett. 77, 4887 (1996).

15. R. Omnès, Understanding Quantum Mechanics, Princeton University Press (1999).

16. R. Omnès, Philosophie de la science contemporaine, Gallimard (1994), English translation by A. Sangalli with additions in Quantum Philosophy, Princeton University Press (1999). 
three directions are furthermore necessarily identica ${ }^{17}$. There could be a nice Kantian echo when this result is expressed as follows: the quantum thing-in-itself has no specific direction of time but pure reason, i.e. logic, can only give an account of it by selecting once and for all a unique direction. Think of it: the direction of time as a categorical a priori judgement !

Decoherence is closely related with wave packet reduction, but they are not identical. Decoherence is a genuine quantum effect occurring inside a measurement device, whereas reduction was supposed to affect directly the measured quantum object and was at variance with the Schrödinger equation. Their statistical consequences are however identical, because decoherence implies that one can compute the probability for the results of a second measurement as if there had been reduction in a previous measurement (this result being most easily shown by using histories). But there was another aspect to reduction. It was also supposed to insure the uniqueness of the measurement result, by selecting a single outcome among various possible ones; it explained, or at least it preserved the uniqueness of reality by an actualising one possibility among many. Decoherence on the other hand performs only the first step of the process. When acting on the environment wave functions (or rather on the state operator), it removes superpositions, entanglements, and leaves only ordinary, classical, probabilities for the various possible results. Being however a quantum effect, it cannot go further and cannot explain how a specific result is selected as the actual one.

It seems at this juncture that we are left again with a very old problem, though now it is rather differently stated, a problem one may call that of actualisation or "objectification". When naively stated, it amounts to the question: is there a genuine effect enforcing actuality ? Less naively, it becomes much more subtle because one cannot even state it as a problem in the framework of quantum logic. This logic implies, indeed, that the only logically consistent histories are the ones referring to a unique result, whatever it may be, and the problem of actuality asking "which one ?" has no content in the theory.

The question (if not the problem) is made deeper because of some new powerful results. Not only is the theory of measurement becoming a collection of theorems, but classical logic and even common sense can be deduced from the quantum principles, in a macroscopic situation. One may then confidently assert that everything observed has been proved to be a direct consequence of these principles ! Everything? Well, there is still this question about uniqueness for which a genuine probabilistic theory cannot obviously provide any cause or mechanism. But if one asks philosophers what is the most essential feature of reality, they say: uniqueness. This is at least what comes out of Wittgenstein's games of language when the apprentice does not understand the word "stone" or "brick" and the sole resource of the master mason is to point out a real stone and say: "that".

17. R. Omnès, Understanding Quantum Mechanics, Princeton University Press (1999). 
"That" is meaningful because and only because "that" is unique. We are thus left with a theory agreeing with every feature of reality, except one, but it is essential. My own belief is that we can learn much more about ontology by studying this question deeper, since it is a pure case of the relation between thought and reality, theory and actuality, mathematics and physics. One might try of course to get out of it through Everett's many-sheeted reality, but it means that one believes quantum theory above the unique wonder of a reality we can contemplate everyday. It looks to me as the extreme of ideology and I would rather prefer bishop Berkeley's unique dream of reality by God, if things have to go that far.

Let us go back however to less elevated questions. I did not yet mention that decoherence is a dynamical effect that is never perfectly exact. Entangled states of a measured quantum object and a measuring device are disentangled, but a tiny amount of entanglement (or superposition) always survives. The probability for observing a macroscopic interference effect between a dead and a live cat is never exactly zero, but extremely small and becoming exponentially smaller with larger values of time. As a matter of fact, very small probabilities pop up everywhere in the new interpretation: in determinism, which is a logical equivalence between two classical properties holding at different times, and which has always a tiny probability for being wrong (because of gigantic quantum fluctuations) ; in the expression of classical properties, which are always slightly spoiled by the uncertainty relations and in other places we can leave out. Borrowing a famous expression from antique philosophy, we might say with Simplicius and St Augustine that quantum theory preserves every appearance of reality, except for extremely small probabilities for having them spoiled. This is again a question about the exact meaning of a physical theory, and certainly the oldest question of that sort.

One may look more carefully at the question of very small probabilities. It is often said that are negligible, but what is the exact meaning or the precise evaluation of "very small"? We might say: a probability that cannot be checked experimentally, even if the measuring device contained all the matter in the Universe (though excessive, this is at leas a "pragmatic" definition and not so crazy when considering that this kind of probabilities always involves an exponential). The next question is then: if our theory agrees with a primary, intuitive, classical experience of the world through our senses (phenomena), except for very small probabilities of error or misconception, can we neglect safely these probabilities and on which ontological grounds?

The first person to ask this question was $f$ mile Borel, the famous mathematician and probabilist. He asked it when thinking of quantum theory in the late thirties and early forties and his answer, which I endorse, can be summarised as $\operatorname{such}^{18}$ : An interpretation of probability calculus must be decided before any interpretation of quantum mechanics,

18. E. Borel, Valeur pratique et philosophie des probabilités, Gauthier-Villars, Paris (1937) ; Le jeu, la chance et les théories scientifiques modernes, Gallimard, Paris (1941). 
since the second theory relies conceptually on the first one. The existence of too small probabilities cannot be falsified by any experiment (in the sense of Popper's notion) ; the corresponding "strange events" or "miracles" (like the Earth leaving the neighbourhood of the Sun to go revolving around Sirius after a tunnel effect or a dead cat coming back to life) are not of course reproducible and therefore, again, their probability has no scientific meaning. Borel went even as far as stating as an "Axiom Zero" of probability calculus that events with too small a probability should be considered as never occurring.

I do not wish to conclude hastily on the fascinating ontological questions we are now discovering with the new data and theoretical results on the foundations of quantum mechanics. Consider how classical concepts are found to emerge from quantum laws; how common sense stands out as a special case of quantum logic, when applied to macroscopic objects and beings; how causality and locality are found as standard consequences of quanta, although they are not universal principles; how very small probabilities would seem to turn reality into an appearance, and nevertheless are so small that unreality does not matter; how quantum theory agrees with everything observed, except for a uniqueness that is the very essence of reality; how we must therefore reconsider the meaning of the Cartesian project in which all of Nature is supposed to be mathematically expressed ; what are then the consequences for the ontological status of mathematics; what could be changed and what should remain if a breakthrough occurred on the frontiers of quantum theory and, most probably, of general relativity. We need bold and careful philosophers for helping us to see more clearly and surely through that wonderful maze. But it cannot be done by cooking again the old meal in old pans. 